\title{
The Comparison between Traditional and Interferometric Multibeam Systems
}

\author{
Kai Ma ${ }^{a^{*}}$, Weiming $\mathrm{Xu}^{\mathrm{b}}$ and Jian $\mathrm{Xu}^{\mathrm{c}}$ \\ Department of Hydrography and Cartography, Dalian Naval Academy \\ 116018, Dalian, Liaoning, P. R. China \\ a869834988@qq.com, bxwm05@mails.tsinghua.edu.cn, cxujian1220@163.com
}

Keywords: Interferometric multibeam; Data density; Horizontal resolution; Data quality

\begin{abstract}
The multibeam system was the integration of acoustic sounding, computers processing, satellite positioning, digital sensors and other technology equipment, had played an important role in the depth measurement. This paper discussed the differences in data density, the precision of the horizontal resolution, and the data quality obtained by the central beams between the interferometric multibeam Geo Swath Plus and the traditional multibeam Sonic2022, by which some useful conclusions were achieved.
\end{abstract}

\section{Introduction}

Multibeam exploration technology rose in the 1970s, and developed rapidly in the 1990s, which was a new kind of submarine topography exploration technology with a high degree of precision. Because the multibeam systems used orientable or non-orientable wide angle launch technology, multichannel reception technology, and the new type acoustic sensor. The hundreds of high-precision banded submarine topography data could be received by this system. Therefore, the multibeam systems had changed the basic concept of old exploration technology, and made the exploration's principle, method, equipment, and data processing, which were different from the traditional acoustic sensor system [1]. During the development of the multibeam systems, there were two kinds of mutltibeam systems with different beam theory. One used beam forming control technology, which was named of the traditional multibeam system, the other used interferometry technology, which was named of the interferometric multibeam system. This paper discussed the differences in data density, the precision of the horizontal resolution, the data quality obtained by the central beams between the interferometric multibeam Geo Swath Plus and the traditional multibeam Sonic2022.

\section{Beam Principle}

The rationale of the multibeam forming is that calculating the cross range and depth by selecting different beams. Whether they are real beams or virtual beams, all the multibeam systems calculate the cross range and depth by measuring following two variables [2].

(i)The distance between the transduser and the measuring point.

(ii)The acoustic path angle from the transduser to the measuring point.

All multibeam systems use one or two of following methods to measure the variables. Method No.1: measuring the time of round-trip signal at a specific angle[3]. Method No.2: measuring the angle of reflected signal at a specific time.

The traditional multibeam system, this kind of multibeam system uses the method No.1 which is called beam forming control technology. The traditional multibeam system receives the directional echo beams and then analyzes the signal of echo to get the acoustic path angle. The depth of the target point can be calculated when the acoustic path angle $\theta$ is achieved as well as the travel time $t$ of the beams.

Based on the first order approximation, the vertical distance $\mathrm{D}$ and the horizontal distance $\mathrm{H}$ can be written by 


$$
\begin{aligned}
& D=v t \cos \theta \\
& H=v t \sin \theta
\end{aligned}
$$

Where $D$ means the vertical distance between the transduser and the seabed, and $H$ means the distance between the measuring point and the projective point of the transduser on the seabed.

To the Interferometric multibeam systems, these kind of multibeam systems use interferometry technology [4]. After scanning the seabed by using short pulse, we can receive a series of echo signals as the particular phases. Based on interferometry principle, there is a relationship between the phase difference and the reflected beam angle of the adjacent tranduser element, the angle can be calculated [5]. And then, the depth of measurement point can be calculated after compensating the roll.

Geo Swath Plus is a typical Interferometric multibeam system, it can transmit acoustic beams alternately by two transduser and receive echo acoustic beams continuously. A series of phase differences of echo beams can be recorded by Geo Swath plus, and a series of angles of reflected beams also can be calculated [6]. The following Fig. 1 shows the geometrical relationship between the tranduser and a seabed point.

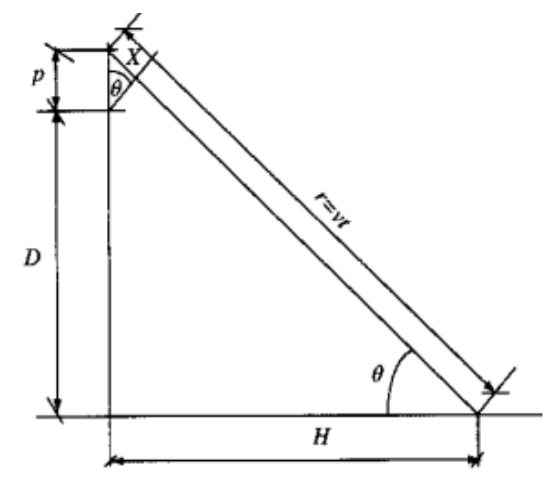

Figure 1. Geometrical relationship between the tranduser

And a measurement point on the seabed

If the phase difference is $\Phi$,wave length is $\lambda$, their relationship is

$X / \lambda=\Phi / 2 \pi$.

Where $X=\mathrm{d} \sin \theta$, and $\Phi=2 \pi \mathrm{d} \sin \theta / \lambda$. the angle $\theta$ of the reflected beam can be obtained[7].The distance between the two adjacent tranduser elements is $p$, the relationship between $\theta$ and $p$ is

$\theta=\arcsin (\Phi \lambda / 2 \pi p)$

From the Eq.4 we can know that the $\theta$ can be calculated by the $\Phi$ and the p. The slant distance $r$ can be written by

$r=v t$.

Where $\mathrm{v}$ means sound velocity and $\mathrm{t}$ means time of sound. If the $\mathrm{p}$ be ignored, the vertical distance $\mathrm{D}$ and the horizontal distance $\mathrm{H}$ also can be written by

$$
\begin{aligned}
& D=r \cos \theta . \\
& H=r \sin \theta .
\end{aligned}
$$

\section{Performance Comparison}

Differences of Data Density. The data density of multibeam systems depends on different physical variables. Traditional multibeam systems data quantities obtained by a single scan are subject to the 
beam angle, and it's actually subject to the quantities of receiver element [8]. The general number of traditional multibeam system receiver elements is 100, It's obvious, the number of receiver elements restricts the data density.

The interferometric multibeam system data volume obtained by a single scan depends on the time interval quantity of the reflected beams received by transdusers. The time interval is generally $50 \mu \mathrm{s}$. Because interferometric multibeam system needn't rely on the quantity of receiver elements, the quantity of interferometric multibeam system is ten even hundreds times as much as the quantity of traditional muitibeam system. It's shown in table 1.

Table 1 comparison of data quantity between two kinds

multibeam system.

\begin{tabular}{lll}
\hline Sensor type & Data quantity $(20 \mathrm{~m})$ & Data quantity $(100 \mathrm{~m})$ \\
\hline Sonic 2022 & $256(\max )$ & $256(\max )$ \\
Geo Swath Plus & 2500 & 12500 \\
\hline
\end{tabular}

Resolution Difference. Data resolution also can be called footprint, for traditional multiubeam system. The footprint tendency of the traditional multibeam system is tangent when the acoustic path angle increases. For example, if the water depth is $20 \mathrm{~m}$, the footprint of 1.5 degrees beam is $0.5 \mathrm{~m}$ when the acoustic path angle is 0 degree, and the footprint of the same beam can be $7 \mathrm{~m}$ when the acoustic path angle is 70 degrees. We can only get one depth value in a footprint, so the error value will be too large if the acoustic path angle over 45 degrees.

The interferometric multibeam system Geo Swath plus uses interferometry technology, it has 4 echo receiver boards, every board can distinguish 1.5 degrees phase [9]. When the four boards work together, the system can distinguish 0.04 degrees phases. The depth resolution of Geo Swath can be $3 \mathrm{~mm}(250 \mathrm{k})$ and $6 \mathrm{~mm}(125 \mathrm{k})$, the lateral resolution of Geo Swath can be $12 \mathrm{~mm}(250 \mathrm{k})$ and $24 \mathrm{~mm}(125 \mathrm{k})$.

Center Beams Issue. About the interferometric multibeam system Geo Swath plus, the differences of the acoustic-paths that are just below the transduser are very small, it can influence the density and the accuracy of the data, so there is a huge difference of the data quality in the central area and the two sides area where the beams irradiate. It's easy to produce bulges or depressions in the process of data processing. Geo Swath Plus set a single beam instrument in the middle of "V" type transduser, in order to check the data in the central area and make up for the data precision.

Because the center beams of Sonic 2022 are concentrated, the traditional multibeam system Sonic 2022 has the small footprints in the central area where the beams irradiate. Sonic 2022 combines two methods to detect the beams, one is named amplitude detection and the other named phase detection [4].This design can complement each other method and operate seamlessly. So there was no sudden change in the data of Sonic 2022 in the central area.

As the Fig. 2 and the Fig. 3 show, they are 3D illumination maps of Geo Swath plus and Sonic 2022 in the same seabed area. Geo Swath Plus has the advantage in resolution, but the points data that are obtained under the central beams have sudden changes [10].We can see the bulge in the Fig. 2, and Sonic 2022 doesn't have this problem. Although Geo Swath Plus use single beam instrument to fill the gap, it also asked for higher standard to operator during data processing.

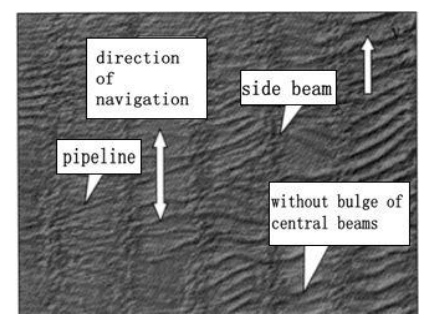

Figure 2. Geo Swath plus 3D illumination map

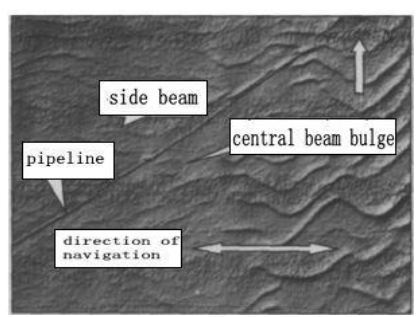

Figure 3. sonic 2022 3D illumination map 


\section{Conclusion}

As show above, we discussed the difference in data density, horizontal resolution of the central beams points accuracy and central beam issue. We can draw the following conclusions.

(1) Interferometric multibeam systems have the advantages in data density and horizontal resolutions over traditional multibeam systems Interferometry technology can help multibeam systems combine with scan sonar.

(2) Because the limitation of the interferometry technology, the points data that are obtained under the central beams have defects. Although the interferometric multibeam systems can add single beam systems to solve the problem, it also increase cost and make systems more complicated to operator.

(3) The interferometric multibeam systems need more memory space than the traditional multibeam systems, it put forward higher requirements on storage device, data processing and modeling algorithm.

\section{Acknowledgements}

This work was supported by the National Science Foundation under Grant 61071006, by the National Science Foundation for Post-doctoral Scientists of China (Grant No.22012T5087, 20110490189), by Open Fund of State Key Laboratory of Information Engineering in Surveying, Mapping and Remote Sensing under Grant 10P03, and by the Start Fund of Liaoning Province Doctor under Grant 20101130.

\section{References}

[1] Peter Hogarth. Shallow Water Surveys Using the Geo Acoustics Geo Swath [M]. Shallow Water Conference-Australia, 2003.

[2] Andy Talbot. Shallow Survey 2005 common data set comparisons [R]. UK: Hydrogr Office, 2005.

[3] L-3 COMMUNICATIONS ELAC NAUTIK. Technical description of multibeam echo sounder system. SEA.BEAM 1180[R]. Kiel: L-3 COMMUNICATIONS ELAC Nautik, 1999:3-9.

[4] IHO Committee. Manual on Hydrography[M]. Monaco: International Hydrographic Bureau, 2005

[5] IHO Standards for Hydrographic Surveys, Special Publication No.44[S]. 4th. IHydrographic Bureau, 1996.

[6] Wessel P, Smith W H F. The Generic Mapping Tools Technical Reference and Cookbook[EB/OL] http://gmt.soest.hawaii.edu/, 2008.4.10.

[7] Mallace D, Robertson P. Alternative Use of CUBE: How to Fit a Square Peg in a Round Hole[C]. U.S. Hydrographic Conference. Norfolk, Virginia: 2007.

[8] Eeg J. On the identification of spiks in soundings [J]. International Hydrographic Review, 1995, 72(1): 33-41.

[9] Geng X, Zielinski A. Precise Muitibeam Acoustic Bathymetry [J]. Marine Geodesy, 1999, 22(3):157-167.

[10] Calder B, Mayer L A. Robust Automatic Multibeam Bathymetric Processing[C]. U. S. Hydrographic Conference. Norfolk, VA, USA: 2001. 